\title{
Automatic serial diluting: an instrument for use in bacteriological laboratories ${ }^{1}$
}

\author{
R. E. TROTMAN \\ From the Wright-Fleming Institute, St. Mary's Hospital Medical School, London
}

SYNOPSIS A machine for automatic serial diluting in bacteriological laboratories is described. It is simple to operate, not too expensive, and results are sufficiently accurate for use in a diagnostic laboratory.

Automatic methods have hitherto been very little used in diagnostic bacteriology, despite the fact that the work load in many departments has been increasing at a rate of about $10 \%$ per annum. However, the serious shortage of technical staff justifies the design of automatic apparatus that is suitable for processing small numbers of specimens even if it seems, at first, rather expensive. If such apparatus can be assembled from a few basic units, each of which may, in principle, be utilized in a variety of different procedures, it may be possible to produce an economic automatic diagnostic system.

In any equipment of this nature simplicity of operation is essential, even at the cost of complicating the control mechanism, but it is rarely necessary for automatic apparatus to perform its function more quickly than it can be performed by hand; what is important is that the total time the operator spends performing the test be minimal.

For many diagnostic test procedures a very high order of accuracy is not essential, and one should aim at producing as high an order of accuracy as is consistent with the other criteria; in fact, it is likely that automatic equipment can easily be made to give a higher degree of accuracy and reproducibility than is generally achieved in routine diagnostic laboratories.

A broad study of the possibilities of mechanization and automation has been embarked upon, and in the present paper a machine constructed to make serial dilutions, as used in the determination of the minimal inhibitory concentration of an antibiotic or a serum antibiotic level, is described.

${ }^{1}$ The invention is covered by patent application no. $32470 / 66$ and full patent rights have been assigned to the National Research and Development Corporation.

Received for publication 2 January 1967.

\section{METHOD FOR DETERMINATION OF MINIMUM INHIBITORY CONCENTRATION (M.I.C.) OF ANTIBIOTICS}

In this laboratory the M.I.C. is determined by the tube dilution method: by successively diluting $1 \mathrm{ml}$. of antibiotic solution in broth, we produce a series of two-fold falling dilutions and subsequently add a standard inoculum of the bacterium under test to each tube.

There are three stages that could be carried out mechanically; the distribution of the initial $1 \mathrm{ml}$. volumes of diluent into the tubes, the successive dilution steps, and the final addition of culture. In this laboratory, the first stage is performed by an automatic dispenser, based on the peristaltic pump. The apparatus described here performs the second stage and apparatus to perform the third stage is under development.

\section{METHODS FOR TRANSFERRING LIQUIDS}

The efficacy of any apparatus for performing serial dilution depends on the device for transferring the liquid from one tube to another. Two important requirements are that it must be a simple matter to change the pipettes in order that dilutions of a number of different antibiotics can be made in quick succession, and that all parts that come into contact with the liquid can be dismantled, sterilized by heat and re-assembled quickly, without affecting either the calibration or the reproducibility of the volume transferred.

Some manually operated and automatic devices for pipetting liquids are commercially available (Broughton, 1965), and Weitz (1957) published details of a manually operated multi-volume pipetting device. Sequeira (1964) has also produced a similar hand-operated machine. However, it is neither convenient nor economic to incorporate these devices in a multi-volume automatic pipetting device and many of them do not satisfy our requirements. It was decided, therefore, to produce a different system. In principle, this consists of a pipette 


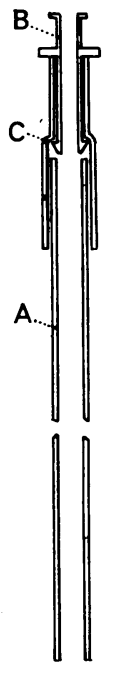

Not drawn to scale.
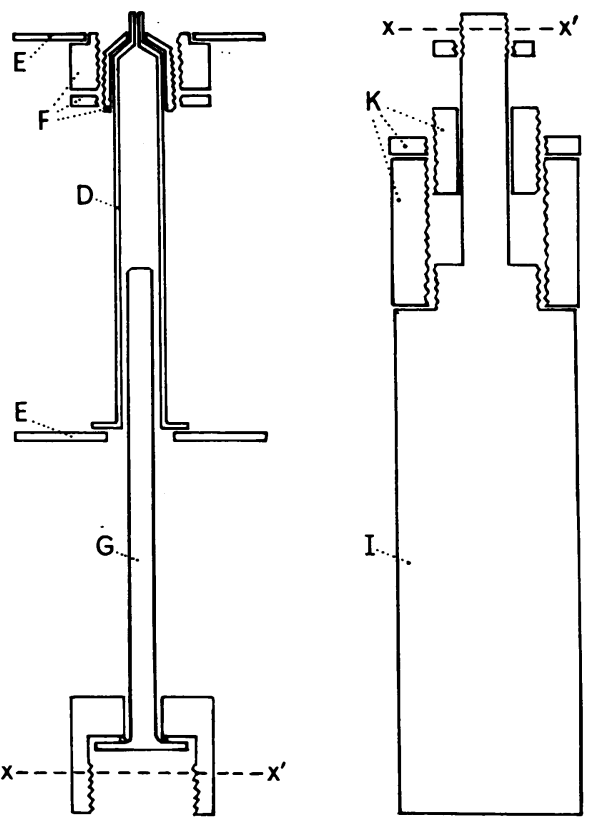

made of a straight length of polytetrafluorethylene (p.t.f.e.) tubing connected by a flexible tube to a $1 \mathrm{ml}$. syringe which is operated mechanically to draw up and expel the fluid. The pipette-syringe unit is mounted in such a way that the pipette can be lowered automatically into the tubes for the dilution and mixing steps.

\section{CONSTRUCTION OF THE DILUTING MACHINE}

THE LIQUID TRANSFER ASSEMBLY (Fig. 1) A syringe is connected, by a Luer connector, to a length of silicone tubing and this in turn is connected to a piece of p.t.f.e. tubing A by a Luer lock connector B. The female part of the Luer lock connector is linked to the p.t.f.e. tube by a short length of silicone tubing $C$. The syringe barrel $D$ is mounted between two fixed brackets $E$, with the aid of device $F$ which is adjustable to allow for tolerances in the external dimensions. The syringe piston $G$ is connected to a 1 in. diameter double-acting pneumatic cylinder $I$, the inward stroke length being varied by device $\mathrm{K}$.

The p.t.f.e. tube, which has a $\frac{1}{8}$ in. internal diameter and $\mathrm{a} \frac{3}{16}$ in. outside diameter, is approximately $6 \frac{1}{2}$ in. long, so that $1 \mathrm{ml}$. occupies approximately $\frac{3}{4}$ of its length; there is therefore no risk of the fluid being drawn into the flexible connecting tube. By disconnecting the pipette at the Luer lock connector a replacement can be introduced very quickly. Using a Sumit tuberculin syringe, the apparatus repeatedly transfers $1 \mathrm{ml}$. within a tolerance of $\pm 1 \%$.

Four pipette-syringe units have been mounted together (Fig. 2) so that up to four separate series of dilutions can be performed at one time. The four syringes are operated by a single pneumatic cylinder and con-

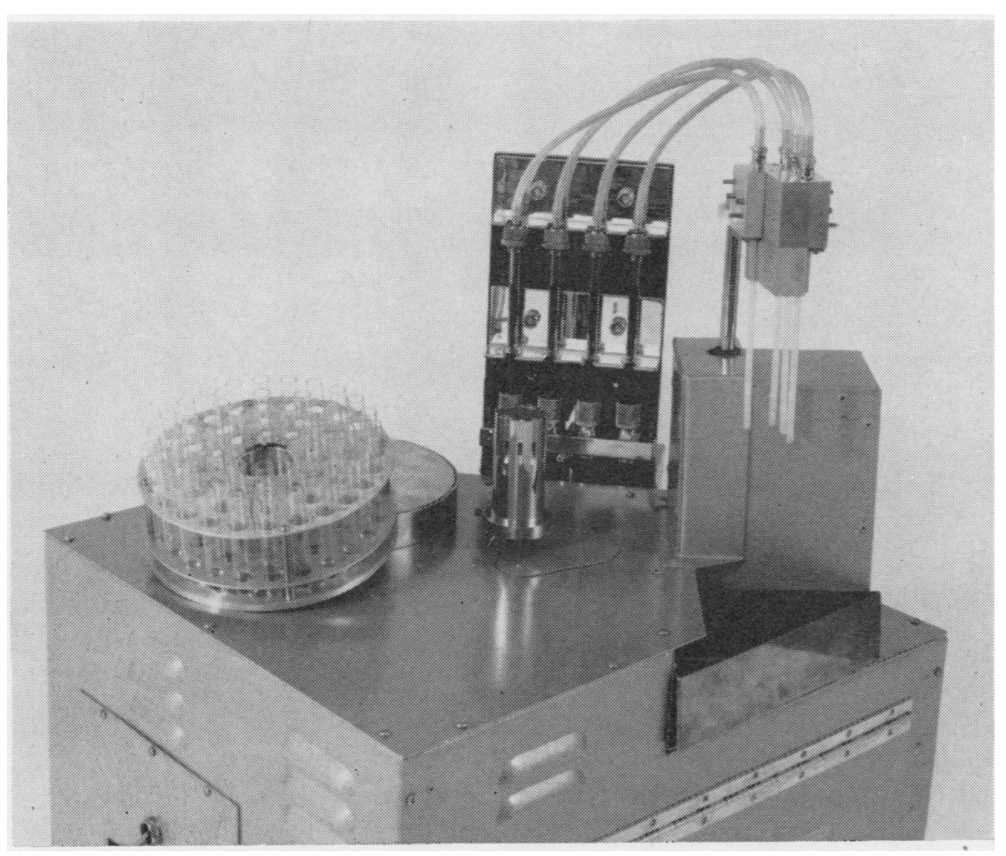

FIG. 2. Photograph of diluting machine showing four syringepipette units mounted together and a rack of dilution tubes. 


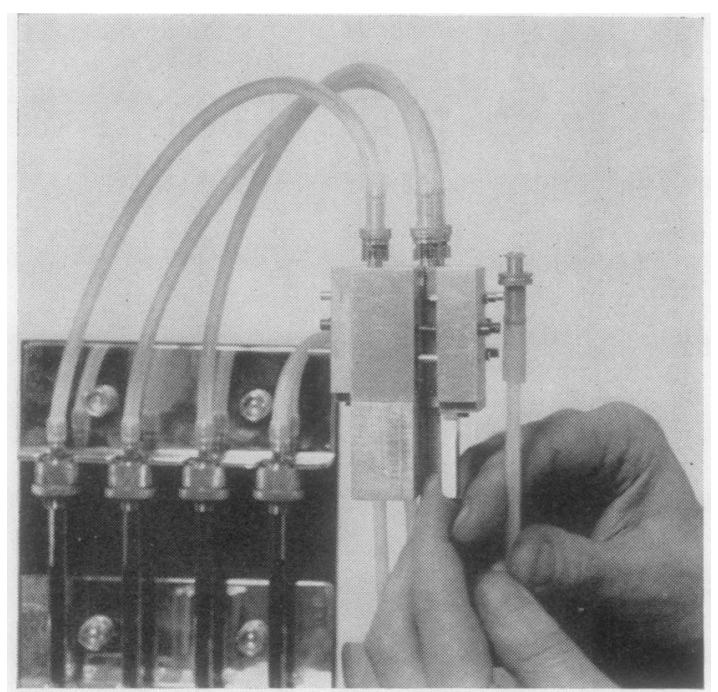

FIG. 3. Close-up view of pipette holder.

sequently the stroke lengths are not independently variable. However, we have found that the syringes in use, which were not especially selected, have a spread in volume transferred of $\pm 0.5 \%$ and so one can quote an overall accuracy of $\pm 2 \%$.

The pipette holder (Fig. 3) is comparatively complicated, but it enables the pipettes to be aligned properly without the operator having to spend a great deal of time checking and altering their positions. It is raised and lowered by means of a second double-acting pneumatic cylinder.

One advantage of a pneumatic cylinder over an electrically driven reciprocating mechanism is that the stroke rate in either direction is independently variable; fine speed control is obtained simply by the turn of a screw thread in a unidirectional air-flow regulator placed in each exhaust air line. This facility is very desirable because one needs to draw up the mixture into the pipette very slowly, in order not to create too much turbulence, to prevent air being sucked up, and to ensure that there is no 'overshoot'. On the other hand, the liquid should be ejected at a much greater rate in order to provide efficient mixing and to ensure that all the liquid is ejected from the tip of the pipette: in some devices the drop left in the tip can be as much as $20 \%$ of the volume transferred.

This is a simple, flexible method of transferring liquids; any small volume may be transferred merely by using an appropriately sized syringe, although if used for volumes smaller than $0.5 \mathrm{ml}$. the accuracy would probably be reduced. The mechanism is so arranged that the meniscus always rises to the same level in the pipette, thus eliminating one source of error often present in manual methods.

The device can simply be made into a heat sterilizable liquid dispenser by attaching a suitable valve mechanism to the nozzle of the syringe. There are many applications for both devices.
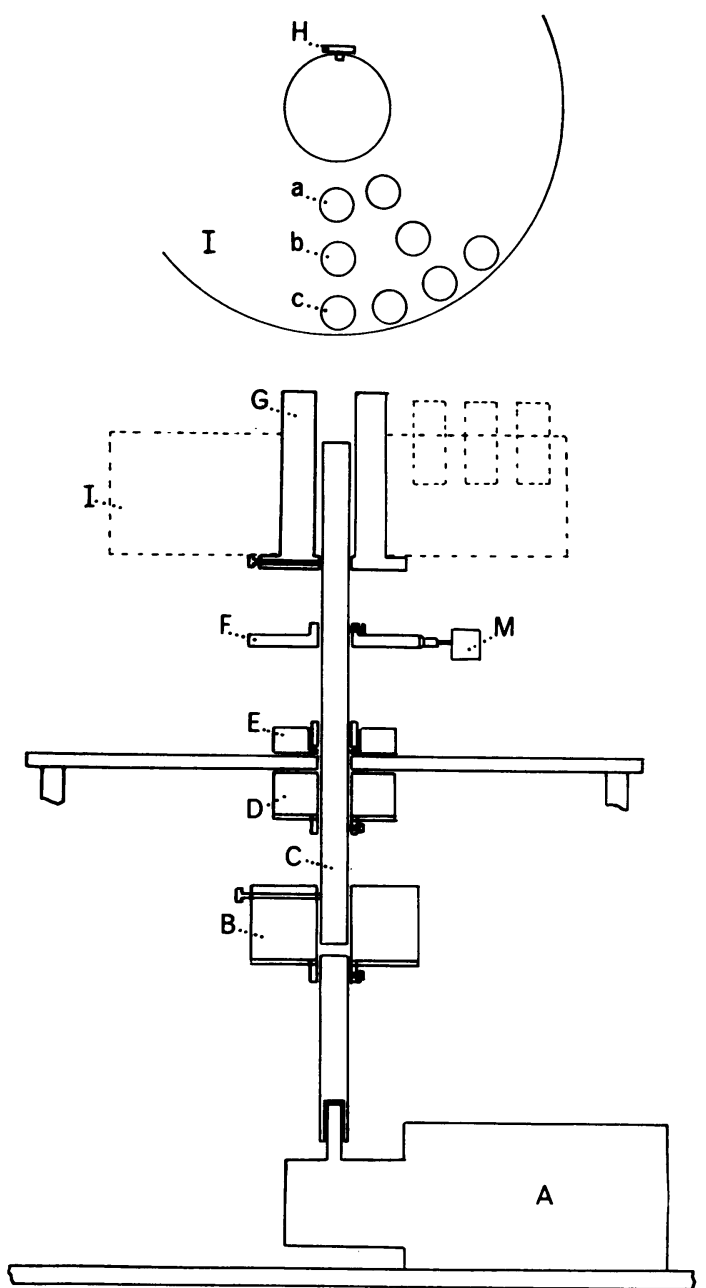

FIG. 4. Drawing of mechanism for automatically feeding the dilution tubes (not to scale). A Induction motor; $B$ electromagnetic clutch; $C$ shaft; D electromagnetic brake; E thrust-race; F grooved disc; $G$ splined rod; $H$ lug; I rack; $M$ microswitch.

AUTOMATIC FEEDING OF DILUTION TUBES There are a number of 'fraction collector' type devices available today but it is surprisingly difficult to find a cheap, compact system that will accommodate only a small number of tubes in a rack that is both small and removable. Consequently it was decided to build one especially.

Figure 4 is a drawing showing the principle used, which is, briefly, as follows. An induction motor A runs continuously, and its shaft is connected to the armature of an electromagnetic clutch $B$. The rotor of the clutch is connected to another shaft $\mathbf{C}$ which runs through an electromagnetic brake $\mathrm{D}$ and thrust-race $\mathrm{E}$. The armature of the brake is attached to this shaft and the fields 
of both clutch and brake are fixed.

A disc with 12 grooves round the circumference, $F$, is mounted on this shaft and actuates a microswitch $\mathbf{M}$ which in turn operates the clutch-brake system in such a way that the shaft will stop at 12 positions per revolution.

Attached to the top of the shaft is a splined rod $G$. There are 12 splines and a lug $\mathrm{H}$ fixed on the racks. I will fit any of the splines, thus enabling the rack to be mounted in any position.

The brake is necessary to prevent the rack overshooting its correct position and to ensure that the splined rod is not rotated when removing or replacing the racks.

Figure 4 shows the layout of the racks; the inner two rows $a$ and $b$ each contain 12 tubes and the outer row $c$ contains 24 tubes. The layout of the pipettes is such that, in effect, we have four series of 12 tubes (or, say, eight series of six tubes). The overall diameter of the rack is 7 in.

This mechanism has a simple basic principle and by varying the number of grooves on disc $F$, the shaft $C$ can be made to stop at any number of positions per revolution; consequently, any number of tubes can be accommodated, facilitating its use in a variety of applications. With a device for moving a dispensing head it makes a versatile fraction collector.

THE COMPLETE APPARATUS Figure 5 shows the complete apparatus. The mechanism for feeding the dilution tubes is mounted in the centre of the right-hand unit and the liquid transferring mechanism is in the right background. On the left-hand side of the motor is the chassis containing the solenoid valves and the unidirectional air-flow regulators that control the pneumatic

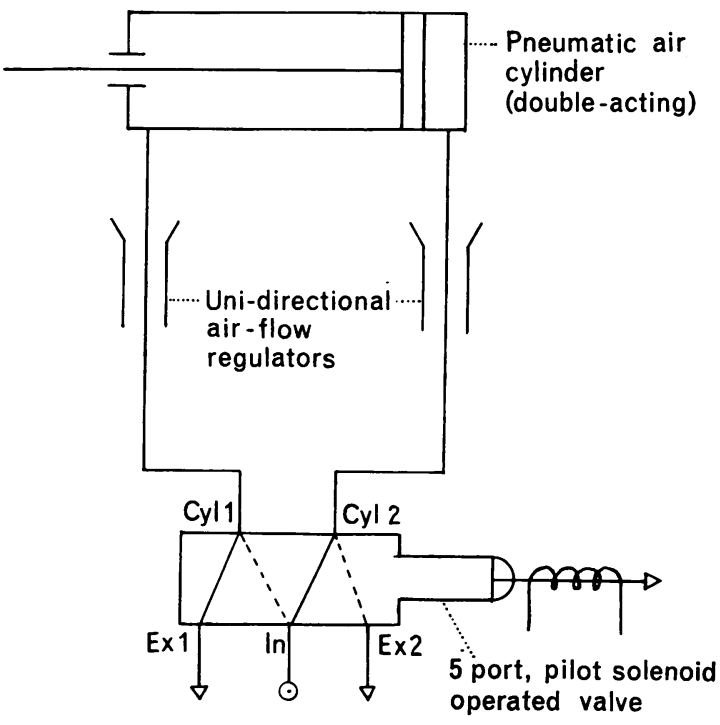

FIG. 6. Circuit diagram of pneumatic air cylinder control system.

cylinders; Fig. 6 is a drawing of the air control circuit for each of the cylinders. In the right foreground (Fig. 5) is the mechanism for raising and lowering the pipette holder.

The left-hand unit is the control system which produces the following sequence of events. On starting, after having placed the prepared rack and pipettes into

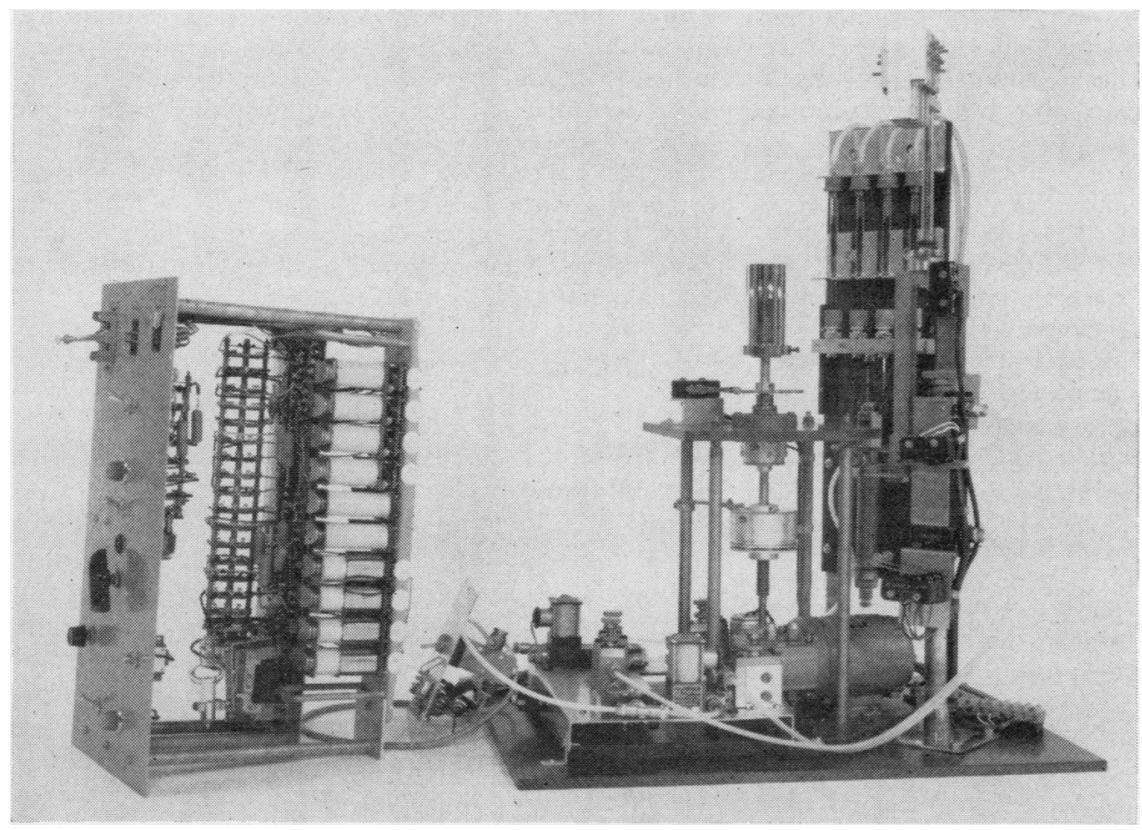

FIG. 5. Photograph of apparatus. 


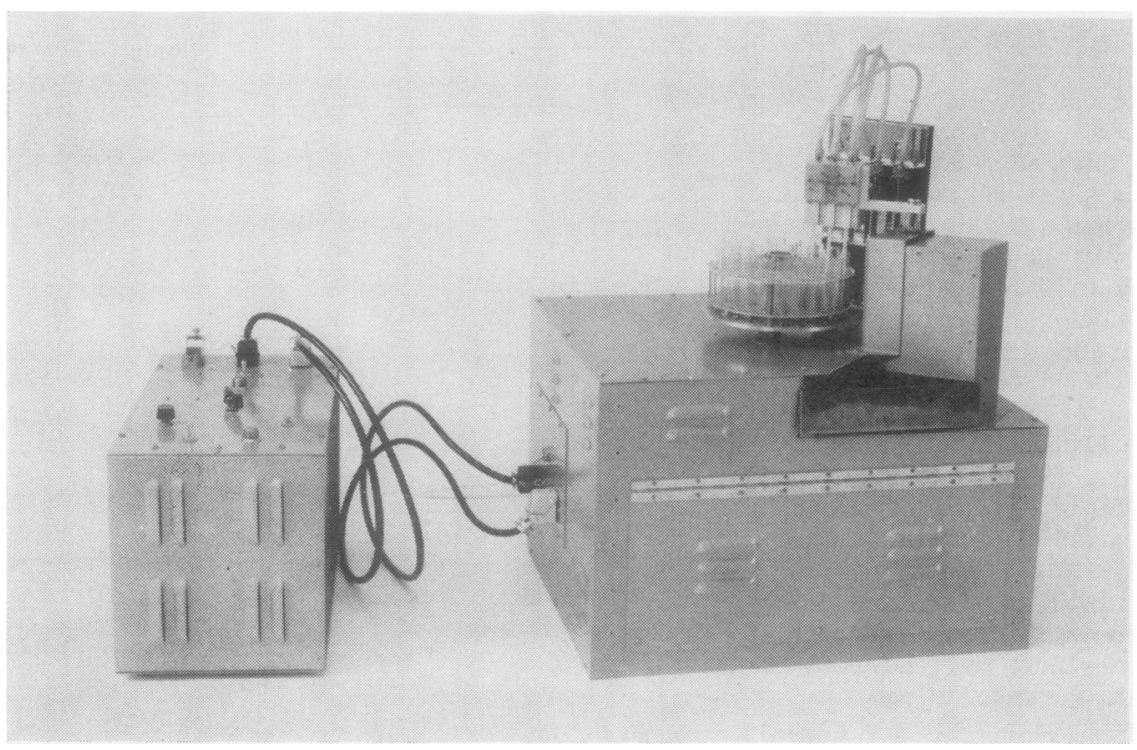

FIG. 7. Photograph of apparatus in use.

position, the pipettes are lowered into the first tube of each series and $1 \mathrm{ml}$. of broth and antibiotic is sucked up into each pipette. After a short delay, this $1 \mathrm{ml}$. is put back into the tube, sucked up and replaced twice and sucked up again. The pipettes are now raised, the rack rotated, the pipettes lowered into the second tube of each series and the procedure is repeated: as no bacteria are present, the same pipettes are used throughout. The number of times that the cycle is repeated is pre-selected by a 12-position switch and the machine stops with the pipettes raised and containing $1 \mathrm{ml}$. of the final dilution. When the pipette holder is rotated in an anticlockwise direction to the position shown in Fig. 2 the final $1 \mathrm{ml}$. is automatically ejected into a waste tray. Figure 7 shows the machine in use.

The instrument is controlled by a sequentially operated electrical system. Post Office electromagnetic relays and solenoid-actuated rotary switches are utilized, together with microswitches which operate when the pistons of the air cylinders reach the end of their strokes. The advantage of the sequentially operated system over a timed sequence is that if one step in not completed properly, the machine will stop. The operator will thus know that something is wrong, which is most important.

It was decided not to use pneumatic control throughout because, first, a counting mechanism is required; secondly, one wanted to keep the demand of air down to a minimum; and, thirdly, this particular sequence of events is more simply achieved by electrical techniques. However, the balance is fairly evenly divided and a pneumatically controlled system has its advantages, particularly if an unlimited supply of compressed air is available.

\section{THE USE OF COMPRESSED AIR}

It may be argued that pneumatic equipment, as used in this apparatus, requires a supply of lubricated com- pressed air that is often not readily available in pathological laboratories, whereas an electrically actuated system would require only the a.c. mains which is invariably available. At first this may appear to be an overriding consideration, but modern compressors are clean, compact and reasonably quiet and it is a simple matter to have one (cost of the order of $£ 150$ ) suitable for operating many pieces of equipment and to pipe the air to various points in the laboratory, as is commonly done in industrial and university laboratories today: if this practice becomes widespread in pathological laboratories, designers of medical laboratory equipment will be able to use, to considerable advantage to all concerned, a vast range of equipment rarely considered hitherto because of lack of air supplies.

We have shown that a practical alternative source of compressed air is the $110 \mathrm{cu}$. ft. cylinder: the machine is used exclusively in the laboratory and there is little difficulty in obtaining and handling the number of cylinders required.

\section{COMPARISON OF PERFORMANCE OF MACHINE WITH MANUAL METHOD}

It is a simple matter to test the accuracy with which $1 \mathrm{ml}$. of liquid is transferred from one dilution tube to the next, but this operation is only one of many possible sources of inaccuracy in the serial dilution technique; poor mixing of the solution and diluent is another obvious source. In order to make a useful comparison between hand-made and machine-made series of dilutions, it was decided to obtain a measure of the concentration of the solution left in each dilution tube at the end of the procedure. We diluted a solution of Armour bovine albumin powder fraction $\mathrm{V}$ in distilled water and measured the optical 
density of each dilution at $2,800 \AA$ on a Unicam S.P.500 spectrophotometer (using $0.5 \mathrm{ml}$. silica cells, a $12 \times 4 \mathrm{~mm}$. slit and collimating lens).

To ensure that any differences in the results were due primarily to those parts of the technique performed by the machine, namely, transferring the solution and mixing it with diluent, very great care was taken to eliminate errors due to inaccurate dispensing of the initial volumes of both solution and diluent. Also, the same initial solution of albumin was used for all tests: inaccuracies in preparing this solution, which are important when an absolute determination of concentration is to be made, are comparatively unimportant when comparing results as here.

It was found to be impractical to measure more than four dilutions of a series because, first, of doubts about the linearity of the calibration curve at intermediate concentrations; secondly, its slope is very small at high concentrations; and, thirdly, the initial solution (dilution 1) would have to be much more highly concentrated and would, therefore, be far too viscous. However, if one obtains satisfactory results for dilutions 3 to 6 , it is reasonable to assume that dilutions 7 to 12 would also be satisfactory, provided the volume of the solution transferred remains within tolerance, as is the case with the machine.

A series of standard solutions, in the range 100 to $1,500 \mu \mathrm{g} . / \mathrm{ml}$., was prepared and the optical densities were measured. With a solution of $4,000 \mu \mathrm{g} . / \mathrm{ml}$. in the first tube (dilution 1), a series of six serial dilutions was produced and then the optical densities of dilutions 3 to 6 were measured. Twelve such series of dilutions were made on the machine and 12 were made manually by members of staff using the technique they normally adopt in their routine work. Thus, we have two sets of 12 optical density measurements for each of dilutions 3 to 6 . In Fig. 8, the highest and lowest values of each set are plotted on the calibration curves. The short vertical lines indicate the calculated concentrations of each dilution. The 12 points for each dilution are not plotted but are fairly evenly distributed between the limiting points.

The points obtained from the machine-made dilutions are more closely bunched together than those obtained from the hand-made dilutions, indicating that the machine produces more consistent results. In the case of dilutions 4 to 6 , the machinemade dilutions are closer to the calculated concentration than those made by hand, indicating that the machine produces more accurate dilutions than those produced manually. Machine-made dilutions 3 appear to be systematically displaced from their calculated positions. However, if the machine introduces a systematic error, dilutions 4 to 6 would be similarly displaced. This is not the case and it is reasonable to conclude, therefore, that these dilutions are more consistent than hand-made dilutions 3 but not significantly more accurate: the displacement is presumably due to a sampling error.

Independent tests to measure the speed and accuracy with which manual operators transfer the solutions from one tube to another showed that the accuracy varied considerably, being as bad as $\pm 7.5 \%$ in one case and as good as approximately

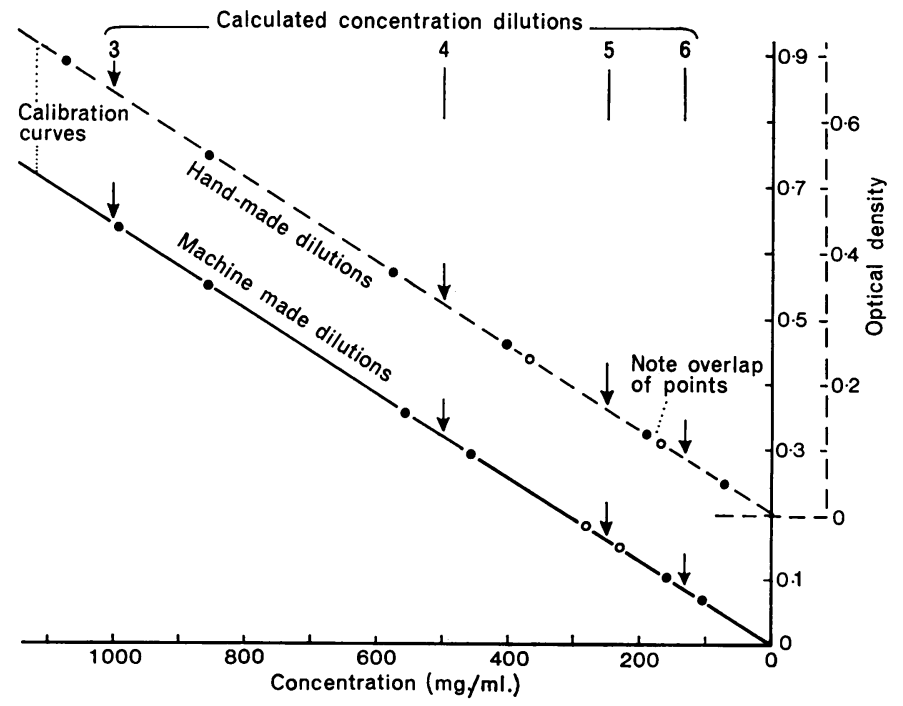

FIG. 8. Graphs showing measured optical densities of hand-made and machine-made dilutions 3 to 6 plotted on identical, but vertically displaced, calibration curves. + , $\times, \bigcirc, \bigcirc-$ measured concentrations ( $\mathrm{mg} . / \mathrm{ml}$.) dilutions 3 to 6 respectively (limiting points only). 
$\pm 1 \%$ in another (Table $I$ ), whereas the machine is consistently within $\pm 2 \%$. This could explain the fact that in the comparative tests described above (Fig. 8), machine-made dilutions 3 were no more accurate than hand-made dilutions 3: hand operators can perform the transfer as accurately as the machine but, being far less consistent, soon make a very inaccurate transfer, thus introducing a large error which may not be nullified.

\section{TABLE I}

\begin{tabular}{|c|c|c|c|c|}
\hline \multirow{4}{*}{$\begin{array}{l}\text { Operator } \\
\text { No. }\end{array}$} & \multicolumn{4}{|c|}{$\begin{array}{l}\text { ACCURACY OF MANUAL AND MACHINE } \\
\text { OPERATIONS COMPARED }\end{array}$} \\
\hline & \multicolumn{2}{|c|}{$\begin{array}{l}\text { Percentage Error in } \\
\text { Volume Transferred }\end{array}$} & \multirow{2}{*}{\multicolumn{2}{|c|}{$\begin{array}{l}\text { Time Taken to } \\
\text { Perform One } \\
\text { Series of } 12 \\
\text { Dilutions }\end{array}$}} \\
\hline & \multirow{2}{*}{$\begin{array}{l}\text { Maximum } \\
\text { Positive }\end{array}$} & \multirow{2}{*}{$\begin{array}{l}\text { Maximum } \\
\text { Negative }\end{array}$} & & \\
\hline & & & Min. & Sec. \\
\hline 1 & 6.9 & $2 \cdot 3$ & 1 & 15 \\
\hline 2 & $2 \cdot 5$ & 1.9 & 1 & 17 \\
\hline 3 & $6 \cdot 0$ & $3 \cdot 2$ & 1 & 17 \\
\hline 4 & 6.5 & $1 \cdot 1$ & 1 & 35 \\
\hline 5 & $7 \cdot 7$ & $2 \cdot 1$ & 2 & 0 \\
\hline 6 & 8.0 & $7 \cdot 4$ & 2 & 0 \\
\hline 7 & $5 \cdot 1$ & $1 \cdot 7$ & 2 & 0 \\
\hline 8 & $6 \cdot 0$ & $4 \cdot 1$ & 2 & 5 \\
\hline 9 & $7 \cdot 6$ & 1.0 & 2 & 5 \\
\hline 10 & $3 \cdot 3$ & $1 \cdot 3$ & 2 & 30 \\
\hline 11 & 5.0 & $1 \cdot 1$ & 2 & 30 \\
\hline 12 & $8 \cdot 7$ & $4 \cdot 5$ & 3 & 0 \\
\hline 13 & $4 \cdot 7$ & $2 \cdot 7$ & 3 & 0 \\
\hline 14 & $3 \cdot 2$ & 1.6 & 3 & 0 \\
\hline 15 & $4 \cdot 2$ & $4 \cdot 7$ & 3 & 5 \\
\hline 16 & $6 \cdot 4$ & $5 \cdot 2$ & 4 & 0 \\
\hline 17 & 0.7 & $1 \cdot 1$ & 4 & 40 \\
\hline 18 & $4 \cdot 8$ & $1 \cdot 1$ & 5 & 0 \\
\hline 19 & $4 \cdot 3$ & $5 \cdot 5$ & 7 & 30 \\
\hline
\end{tabular}

Eleven out of 19 people performed one series of 12 dilutions in under $2 \frac{1}{2}$ minutes (see Table I), the time taken by the machine. The operators knew why they were asked to perform the task and were doubtless trying very hard, and also they performed only one series of dilutions so that the task did not become tedious. It is reasonable, therefore, to assume that this is the best performance they are likely to achieve in the normal course of events. Since the machine performs four series of dilutions simultaneously, it will perform three or four series more quickly than they can be performed manually, assuming the preparative work takes the same time. Considerable saving of time is achieved, however, if five or more series have to be performed, because while the machine is diluting four series more dilution tubes can be prepared.

\section{FINAL COMMENTS}

Although the machine is not very sophisticated, it satisfies the criteria against which we estimate its suitability for use in a diagnostic bacteriology laboratory: it is suitable for processing small numbers of specimens, all parts that come into contact with the liquid can simply be dismantled and sterilized by heat, and it performs a task which is a necessary part of at least two routine diagnostic procedures. Although our embodiment is arranged to transfer $1 \mathrm{ml}$. of solution and to accommodate four series of 12 tubes, it is, in principle, very flexible and it may well find applications in other disciplines.

One may ask if the overall accuracy of the method is adequate. It is possible, of course, to design apparatus that performs each part of the procedure more accurately, or to design apparatus that produces a series of dilutions in such a way that errors are not cumulative. However, in either case, the apparatus would be much more complex and costly, and would probably not be economic in view of the comparatively small numbers of specimens to be processed. In the case of M.I.C.s, it is necessary to decide, therefore, whether the tube dilution method or one of the alternative methods is the most suitable in any given circumstance. If one decides to use the tube dilution method, it is necessary, as Branch, Starkey, and Power (1965) have pointed out, to standardize the procedure. A machine, such as the one described here, could well be the nucleus of such a procedure.

We are greatly indebted to the Trustees of the Nuffield Foundation who are financing the project.

The technical assistance of S. Haskey, G. Dickerson, and J. R. Robinson is gratefully acknowledged.

\section{REFERENCES}

Branch, A., Starkey, D. H., and Power, Edna E. (1965), Appl. Microbiol., 13, 469.

Broughton, P. M. G. (1965). Association of Clinical Biochemists, Technical Bulletin No. 6.

Sequeira, P. J. L. (1964). Personal communication.

Weitz, B. (1957). J. clin. Path., 10, 200. 